\title{
Application of GIS and Statistical Modelling for Dengue Fever Surveillance in Delhi, India
}

\author{
Mahesh Kumar Jat and Shuchi Mala
}

\begin{abstract}
In recent years, Geographic Information System (GIS) has become popular in the field of epidemiology for the identification of unusual spatial patterns of disease occurrences and monitoring. Over the years, global burden of Dengue Fever has increased drastically. According to WHO (Global Environmental Change, Geneva) report, there is a strong indication of inter-annual variability and link between meteorological factors and infectious diseases such as Dengue Fever and many other vector-borne diseases. Recently, researchers have demonstrated the importance of determining the impact of various meteorological factors on the propagation of the infectious diseases and such impacts will be more pronounced in a climate change scenario. To control the spread of any infectious disease, it is crucial to identify areas with higher disease risk. The present work is aimed at using GIS techniques such as Point Density method and Empirical Bayesian Kriging method to produce optimal spatial distribution and prediction of Dengue cases in Delhi using historical Dengue Fever case counts and their geographic locations. Poisson regression method has been used to determine the association of Dengue Fever incidences with selected meteorological parameters such as relative humidity, mean temperature and mean wind speed. The outcomes of this study proved that Dengue Fever occurrences are not random. Also, the regression results revealed that there is a significant relationship between meteorological parameters and Dengue Fever incidences. Thus, providing strong evidence that climatic characteristics are playing an important role in the transmission of Dengue Fever in Delhi in addition other important causative factors.
\end{abstract}

Keywords-Dengue Fever; Significant; Poisson Regression; Geographic Information System

\section{Introduction}

Dengue Fever is found in tropical and sub-tropical regions around the world, predominantly in urban and semi-urban areas[1]. According to the World Health Organization factsheet, few researchers have recently estimated 390 million dengue infections per year, of which 96 million notified clinically. In developing countries such as India, Dengue Fever remains as an important public health concern. As reported by the National Health Profile 2015, total reported dengue cases in India in the year 2014 were 40425 (provisionally). Mosquito-borne disease outbreaks occur primarily in areas where mosquitoes that carry a Dengue, Chikungunya or Zika virus live.

Mahesh Kumar Jat, Associate Professor and Shuchi Mala, Research Scholar

Department of Civil Engineering, Malaviya National Institute of Technology Jaipur, Rajasthan, India
These pathogens are present in mosquitoes and hosts that interact in spatially heterogeneous environments where hosts travel between regions of varying transmission intensity[2]. The geographic distribution of these diseases are shifting because of vector control, economic development, urbanization, climate change, land-use change, human mobility, and vector range expansion [3], [4]. Therefore, to get a deeper understanding of spatiotemporal dynamics of these diseases, GIS plays an important role. Understanding the space-time distribution of mosquito-borne disease like Dengue Fever is essential in the planning and implementing of effective disease control measures[5]-[11].

Geographic Information System provides a platform for integration of various diseases related information, which may be collected from diverse sources in different formats and scale, into a standardized spatio-temporal information and this can help in understanding occurrences, distribution of diseases and their relationship with surrounding environment (socioeconomic conditions, environmental and meteorological characteristics).

Spatial interpolation methods such as Empirical Bayesian kriging $(\mathrm{EBK})$ is a geostatistical interpolation method that automates the most difficult aspects of building a valid kriging model[12]. The main advantage of EBK is that, it automatically determine the parameters such as transformation type, decluster before transformation and order of trend removal through a process of sub-setting and simulations while other kriging methods require manual adjustment of parameters to receive accurate results. In our analysis, result of EBK provides the clear distribution of disease incidences which may help in identifying sensitive areas of disease occurrences. Point Density method calculates the density of point features around each output raster cell. A neighborhood is defined around each raster cell centre, and the number of points that fall within the neighborhood is totalled and divided by the area of the neighbourhood. The result of point density analysis provides us deeper insight about the influence of disease outbreaks in one location to the neighbouring locations. To determine the impact of meteorological parameters on the occurrence of disease outbreaks, regression techniques such as Poisson regression and Logistic regression are used. The basic idea of Poisson regression was outlined by Coleman [13] and this method is used to model count data. Poisson regression models the (natural) log of the expected count. The results of Poisson regression helps to identify positive, negative or no association between the dependent variable that is number of cases and independent variables such as relative humidity, mean temperature and mean wind speed. 
Until now, all research has focused on biological, entomological and clinical aspects of Dengue Fever (DF)/ Dengue Hemorrhagic fever (DHF)/ Dengue Shock Syndrome (DSS) separately[1]. Few researchers have reported the relationship between number of dengue virus infections and climatic factors using several indices and statistical techniques, and it has been concluded that climatic conditions have a strong influence on vectorborne infection[3], [8], [11], [14]-[16]. Very limited research has been done to identify the impact of sociocultural practices and environmental factors on the DF incidences in India[5], [17]-[19]. There is a strong need to understand the interrelationship of socio-cultural practices and environmental factors with vector-borne disease incidences using statistical modelling and Geographic Information System.

Therefore, the present study is aimed at using Empirical Bayesian Kriging method to understand spatial variation in the occurrence of Dengue Fever incidences, a vector borne disease in Civil Lines and Karol Bagh Zones of Delhi, capital city of India, for the study period 2011 to 2012. Also, an attempt has been made to identify the areas where the outbreak intensity is high by using Point Density method. Poisson regression technique has been used to determine important meteorological parameters (relative humidity, mean temperature and mean wind speed) responsible for high DF incidences.

\section{Materials and Methods}

\section{A. Study Area}

Delhi is the National Capital of India. According to the census report, it has a population of about 16.3 million. Delhi has typical version of the humid subtropical climate. Temperature in Delhi usually ranges from 5 to $40{ }^{\circ} \mathrm{C}$ with the lowest and highest temperatures ever recorded being 6.7 and $47.8^{\circ} \mathrm{C}$. The monsoon starts in late June and lasts until mid-September, with about $797.3 \mathrm{~mm}$. The entire Municipal Corporation of Delhi (MCD) area is divided into 12 zones spread through three smaller municipal corporations which are North Delhi Municipal, Corporation South Delhi Municipal, Corporation and East Delhi Municipal Corporation as shown in Figure 3.

\section{B. Data Sources}

Daily cases of DF from year 2011 to year 2012 have been taken for the study area from Health Department of Civil Lines and Karol Bagh Zone of Municipal Corporation of Delhi (MCD). MCD is a government agency of India which brings together confirmed cases of epidemics collected from the different hospitals. In this paper, analysis is carried out at local level. In this analysis, the details of dengue cases have been taken from Epidemiological Forms filled at the time of treatment of patients. The data included the date, residential location, age and gender of the notified cases of DF. Data related to meteorological parameters such as relative humidity, mean temperature and mean wind speed have been taken from India Meteorological Department (IMD).

\section{Spatial Analysis using Empirical Bayesian Kriging method}

Empirical Bayesian Kriging method is different from classical kriging methods by accounting for the error introduced by estimating the semivariogram model [12]. Semivariogram is defined in equation 1 where var is the variance, $s_{i}$ and $s_{j}$ are two locations and $Z\left(s_{i}\right)-Z\left(s_{j}\right)$ is the difference in their values. If $s_{i}$ and $s_{j}$, are close to each other in terms of the distance measure of $d\left(s_{i}, s_{j}\right)$, these are expected to be similar, so the difference in their values, $\mathrm{Z}\left(\mathrm{s}_{\mathrm{i}}\right)-\mathrm{Z}\left(\mathrm{s}_{\mathrm{j}}\right)$, will be small. As $\mathrm{s}_{\mathrm{i}}$ and $\mathrm{s}_{\mathrm{j}}$ get farther apart, they become less similar and difference in their values, $\mathrm{Z}\left(\mathrm{s}_{\mathrm{i}}\right)-\mathrm{Z}\left(\mathrm{s}_{\mathrm{j}}\right)$, will become larger. This method comprised of three main steps which are: firstly, a semivariogram model has been estimated from the data. Secondly, this semivariogram has been used to simulate a new value at each of the input data locations. Lastly, new semivariogram model has been estimated from the simulated data. Bayes' rule has been used to calculate weights for this semivariogram, which shows how likely the observed data can be generated from the semivariogram. Further, a new semivariogram model and weights have been estimated using simulated data. Predictions and prediction standard errors are then calculated at the unsampled locations using these weights. Each semivariogram is an assessment of the true semivariogram from which the observed process could be produced [12].

$\gamma\left(\mathbf{s}_{\mathrm{i}}, \mathbf{s}_{\mathrm{j}}\right)=1 / 2 \operatorname{var}\left(\mathrm{Z}\left(\mathbf{s}_{\mathrm{i}}\right)-\mathrm{Z}\left(\mathbf{s}_{\mathrm{j}}\right)\right)$

\section{Point Density}

The purpose of density analysis is to determine the spatial relationship between any phenomenon with the associated factors like relationship between dengue incidences and surrounding causative phenomenon like meteorological characteristics and location of water bodies etc. Point density method calculates a magnitudeper-unit area from point features that fall within a neighbourhood around each cell.

\section{E. Poisson Regression Analysis}

Poisson regression models are generalized linear models used to model count data with the logarithm as the link function and the Poisson distribution function as the assumed probability distribution of the response[20]. For count data $\mathrm{y}_{\mathrm{i}}$ having integer values $\left(\mathrm{x}_{\mathrm{i}}\right)$ like $0,1,2 \ldots$ the model is Poisson regression with parameter $\lambda$ which has been defined as the mean number of occurrences. The general Poisson regression model has been defined in equation 2 [7] where $\beta_{\mathrm{i}}$ are the coefficients of regression, $\mathrm{y}$ is the dependent variable and $\mathrm{x}_{\mathrm{i}}$ are the independent variables.

$E\left[y_{i} \mid x_{i}\right]=\lambda_{i}=\exp \left(x_{i}^{*} \beta\right)=\exp \left(\beta_{1}+\beta_{2} x_{2 i}+., \beta_{k} x_{k i}\right)$ Sometimes, Poisson regression is known as Log-linear model, defined in equation 3 .

$\ln E\left[y_{i} \mid x_{i}\right]=\ln \left(\lambda_{i}\right)=x_{i}^{\prime} \beta=\left(\beta_{1}+\beta_{2} x_{2 i}+. . \beta_{k} x_{k i}\right)$

The interpretation of Poisson regression coefficients are different from that in Ordinary least square model due to exponentiation. According to the results of some calculus and algebra, one unit change in the $\mathrm{j}^{\text {th }}$ regressor leads to a 
change in the conditional mean by the amount $\mathrm{E}\left[\mathrm{y}_{\mathrm{i}} \mid \mathrm{x}_{\mathrm{i}}\right] * \beta_{\mathrm{j}}$ whereas in the linear model it would have simply $\beta \mathrm{j}$. If $Y_{i}$ are independent observations with corresponding values $x_{i}$ of the predictor variables, then $\beta$ can be estimated by maximum likelihood. The likelihood function for a given value is defined in the equation 4 .

$L_{i}=\frac{\lambda_{i}^{y_{i}}}{y_{i} !} e^{-\lambda_{i}}$

Suppose the given data set is containing $\mathrm{n}$ values. Then the likelihood of all the $n$ values is calculated by equation 5. Log likelihood $(1=\log (\mathrm{L}))$ is given by equation 6 .

$L=\prod_{i=1}^{n} L_{i}=\prod_{i=1}^{n} \frac{\lambda_{i}^{y_{i}}}{y_{i} !} e^{-\lambda_{i}}$

$l=\sum_{i=1}^{n} y_{i} \log \left(\lambda_{i}\right)-\lambda_{i}-\log \left(y_{i} !\right)$

To estimate the $\beta$ maximum likelihood estimation is calculated by equation 7,8 and 9 .

$$
\begin{aligned}
& l(\beta)=\sum_{i=1}^{n} y_{i}\left(x_{i}^{\prime} \beta\right)-e^{x_{i}^{\prime} \beta} \\
& \frac{d(\beta)}{d(\beta)}=\sum_{i=1}^{n} x_{i}\left(y_{i}\right)-e^{x_{i}^{\prime} \beta} \cdot x_{i} \\
& \sum_{i=1}^{n} x_{i}\left(y_{i}-\lambda_{i}(\beta)\right)=0
\end{aligned}
$$

Poisson Regression model has various advantages over ordinary linear regression model such as it follows discrete distribution and the restriction of predicted values to non-negative numbers.

\section{Results and Discussion}

\section{A. Point Density Analysis}

A continuous density surface map is created to determine the Dengue Fever density in Delhi region using point density method. The purpose of point density method is to visualize pointwise disease incidences and it helps in identifying areas of high outbreak intensity. The figure 2(a) and 2(b) shows the infection density that is number of disease cases for years 2011-2012. For the year 2011 and 2012 high density areas are Anand Parbat, Kirti Nagar, areas near Prasand Nagar lake and Yamuna river. For the year 2012, more locations are identified having high infection intensity such as KhabasPura, Maulana Azad Medical College Campus. According to the results of the point density analysis, some areas have shown high infection density for the year 2011 and 2012, therefore these areas should be taken into consideration before planning the proactive strategies for the control of Dengue Fever incidences.

\section{B. Empirical Bayesian Kriging method based spatial Analysis}

It is very important to understand the geographic spread of dengue incidences, so that the reasons behind the assemblage of DF in the study area can be determined. Empirical Bayesian Kriging method has been used for this purpose. This method is used to construct a semivariogram, which is a graphical representation of the autocorrelation in a scatter point set, as a function of distance. The kriging method has been implemented in ArcGIS version 10.1 using Geostatistical Analyst (ESRI, Redlands, California) tool. In this method, number of cases at known locations have been used to construct a continuous surface of predicted number of cases at each location. The maximum predicted value that is the number of cases is 6 . The locations with higher predicted value are areas near Maulana Azad Medical College Campus, Bhalswa, KhabasPura, Karawal Village and Palam Village. With the help of predicted number of cases at each location, the spatial pattern of Dengue Fever for the duration 2011-2012 can be analysed and the result has been presented in Figure 3.

\section{Association of Dengue Incidences with Meteorological Parameters}

Poisson regression method has been applied for identifying the association between monthly dengue incidences and meteorological parameters. The standard regression equations where the independent variables are monthly mean wind speed, relative humidity and mean temperature have been shown in equation 10, 11 and 12, respectively. In these equations, $\mathrm{Y}$ is the monthly number of cases, $\mathrm{X}$ is the monthly mean wind speed, $\mathrm{Z}$ is the monthly relative humidity and $\mathrm{W}$ is the monthly mean temperature. To test the statistical significance of the association of Dengue Fever cases with meteorological parameters, p-value has been calculated and standard error has been determined as shown in table 1 . P-value is a function of the observed model results that is used for testing a statistical hypothesis. For our analysis, we have chosen confidence level to be $95 \%$. Before a test is performed, a threshold value is selected which is the significance level of the test. If the $\mathrm{p}$-value is equal or smaller than significance level $(p \leq 0.05)$, it suggests that the observed data is inconsistent with the assumption that the null hypothesis is true and thus it can be rejected. In our case, null hypothesis is defined as there is no association between the Dengue Fever cases and the meteorological parameter.

According to our results, null hypothesis has been rejected and it is found that the influence of mean wind speed is more on the dengue incidences as compared to the influence of relative humidity and mean temperature on the dengue incidences. The primary vector of dengue Aedes aegypti, is highly adapted to all manmade and natural environments and is closely associated with human dwellings[21]. Mosquito-borne disease transmission is closely related to climate because a warm ambient temperature is crucial to the mosquito's life and gonotrophic cycle and virus replication [22].

In this paper, various causes of DF incidences in Delhi have been identified and classified into three categories. Firstly, according to the IMD Monsoon 2012 Report, the monsoon period in Delhi is observed from July to September. During monsoon season, Delhi experiences hot, high to very high humidity and heavy precipitation. During this season, water logging is a very common problem in Delhi due to poor drainage system. Due to water logging, the rate of mosquito breeding increases and therefore more dengue cases have been reported during September to November. Secondly, at higher temperature, Aedes mosquitoes develop from eggs to adults in a shorter period and also experience a shorter incubation period for dengue viruses[23]-[25]. However, the mortality rates of adult mosquitoes increases with 
increasing temperature above $30^{\circ} \mathrm{C}[26]$. According to our analysis, the temperature from July to September for 2011-2012 ranges between $37{ }^{0} \mathrm{C}$ to $25{ }^{\circ} \mathrm{C}$ approx., which is favourable for developing adults Aedes mosquitoes and thus resulting in higher dengue incidences. Also, higher the mean temperature and relative humidity, faster is the vector and pathogen development. Thirdly, lower the mean wind speed, larger the life spans of the vectors. Thus, increasing the dengue incidences. According to some studies, humidity, wind speed and temperature are important meteorological parameters responsible for higher dengue incidences[7], [8], [10], [11], [14], [27], [28],[29]. Therefore, it is very important to understand these associations deeply for developing the control strategies related to vector-borne diseases.

$$
\begin{aligned}
& E[Y \mid X]=\exp (7.4235-0.5712 X) \\
& E[Y \mid Z]=\exp (3.6510+0.0205 \mathrm{Z}) \\
& E[Y \mid W]=\exp (0.9008+0.1714 \mathrm{~W})
\end{aligned}
$$

According to the IPCC 2013 report, climate change is a long-term challenge and it requires critical action by considering the pace and the scale by which greenhouse gases are accumulating in the atmosphere and this increases the risks of a more than 2 degree Celsius temperature rise. The transmission of vector-borne diseases involve a number of factors like some of which will increase transmission as temperatures rise in the near future (decreasing basic incubation periods in the vectors) and some of which will decrease it (increasing vector mortality rates)[30], [31]. Therefore, it is very crucial to determine the exact association of meteorological parameters with vector-borne disease transmission for the prediction of hotspots of an epidemic. The purpose of using Empirical Bayesian Kriging method is to identify spatial patterns of DF occurrences. Analyzing the results, helped to understand the propagation of DF cases spatially. In our analysis, we have used Poisson regression method to identify statistically significant meteorological parameters such as relative humidity, mean temperature and mean wind speed responsible for high DF incidences in Delhi. Thus, providing strong indication that meteorological parameters are playing an important role in the transmission of DF in Delhi.

The advantage of using Empirical Bayesian Kriging method is that it allows accurate predictions of moderately nonstationary data. Also, requires minimum interactive modelling therefore the predicted spatial patterns of the Dengue Fever incidences are more accurate as compared to the other kriging method. With the help of the results of point density method, health priorities areas can be more clearly identified and categorized (extreme priority, high priority and medium priority areas).We have established the association of Dengue Fever cases with meteorological parameters such as relative humidity, mean temperature and mean wind speed using Poisson regression.

The study does have some potential limitations. The length of study period is shorter and no population at risk data is included in the analysis. The study analyzed spatial distribution of DF in Delhi but examined limited causes. Future research should focus on the effect of various socio-economic and environmental factors that could affect disease transmission.

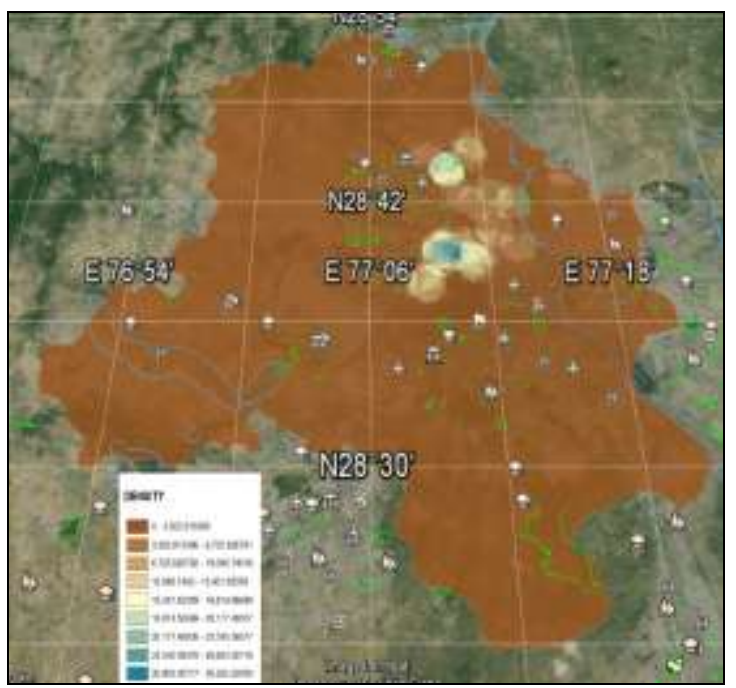

Figure 2(a). Predicted density at Each Location for 2011

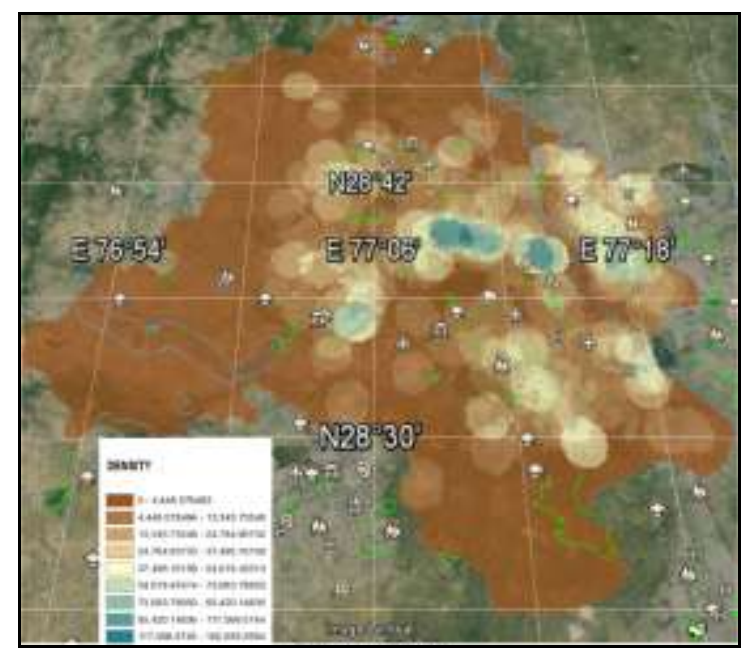

Figure 2(b). Predicted density at Each Location for 2012

Table 1. Showing the results of Poisson regression

\begin{tabular}{|l|l|l|l|l|l|}
\hline Meteorological Parameter & $\begin{array}{l}\text { Regression } \\
\text { Coefficient }\end{array}$ & $\begin{array}{l}\text { Confidence } \\
\text { Interval (95\%) }\end{array}$ & $\begin{array}{l}\text { Standard } \\
\text { Error }\end{array}$ & P-value & $\begin{array}{l}\text { Association with } \\
\text { DF Incidences }\end{array}$ \\
\hline Mean Wind speed( km/hr) & -0.57 & $-0.601,-0.54$ & 0.012 & $<0.05$ & Negative \\
\hline Relative Humidity (\%) & 0.02 & $0.02,0.02$ & 0.002 & $<0.05$ & Weak Positive \\
\hline Mean Temperature $\left({ }^{\circ} \mathrm{C}\right)$ & 0.17 & $0.14,0.19$ & 0.0137 & $<0.05$ & Positive \\
\hline
\end{tabular}


Proc. of the Fourth International Conference on Advances in Civil, Structural and Environmental Engineering - ACSEE 2016. Copyright ( $)$ Institute of Research Engineers and Doctors. All rights reserved.

ISBN: 978-1-63248-114-6 doi: 10.15224/ 978-1-63248-114-6-17

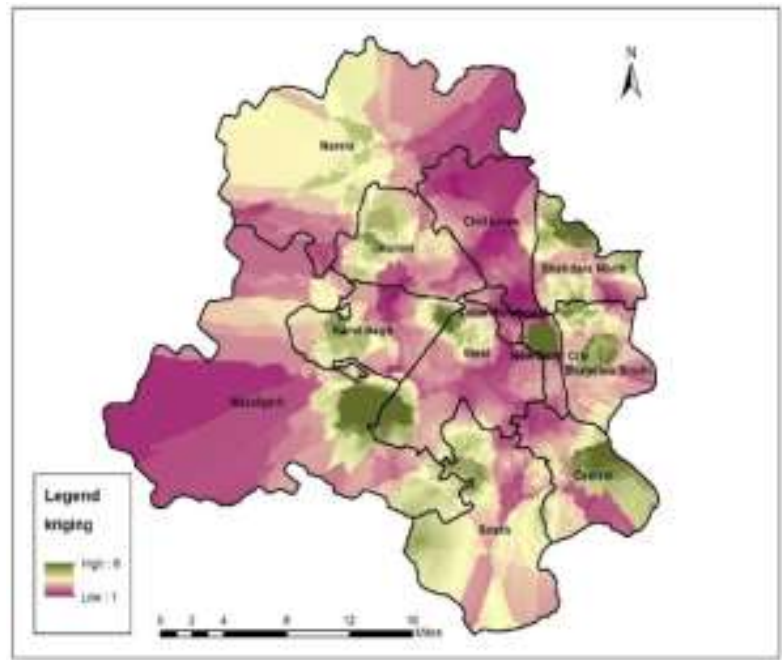

Figure 3. Predicted Number of Cases at Each Location

\section{Iv. Conclusions}

The study has shown the existence of hotspots of DF occurrence for the years 2011-2012, which was very high in Bhalswa, Feroz Shah Kotla, Anand Parbat, Model Town and GTB Hospital Campus of Delhi. We have also shown the spreading of geographic distribution of DF over the study period. Our study contributes to explain the role of relative humidity, mean temperature and mean wind speed in the transmission and distribution patterns of DF cases in Delhi. The results demonstrate the necessity to further improve our understanding of the impact of socio-environmental changes on the transmission of DF. The study has illustrated how, using existing health data and GIS, can provide public health officials with necessary information about the prevalence of statistically significant hotspots of DF in Delhi, thus enabling them to develop more effective strategies to control its spread.

\section{Acknowledgments}

Authors are thankful to Fund for Improvement of S\&T Infrastructure of the Department of Science \& Technology, Government of India for funding the study. Authors are thankful to Dr Ashok Rawat, Deputy Health Officer, Karol Bagh Zone, and Dr. Sanjay Sinha, Deputy Health Officer, Rohini Zone, Health Department, Municipal Corporation of Delhi for providing the data on Dengue Fever incidences to perform the investigation. Author is thankful to Ministry of Human Resource Development, India for providing fellowship to carry out the research work.

\section{References}

[1] K. P. Bhandari, K. Raju, and B. Sokhi, “Application of GIS modeling for Dengue Fever prone area based on socio-cultural and environmental factors-a case study of Delhi city zone," Int Arch Photogramm Remote Sens Spat Inf Sci, 2008.

[2] M. Acevedo, O. Prosper, K. Lopiano, and N. Ruktanonchai, "Spatial Heterogeneity, Host Movement and Mosquito-Borne Disease Transmission," PLoS One, 2015.

[3] K. Nakhapakorn and N. Tripathi, "An information value based analysis of physical and climatic factors affecting Dengue Fever and dengue haemorrhagic fever incidence," International, 2005.

[4] A. Tatem, P. Gething, and D. Smith, "Urbanization and the global malaria recession," Malaria, 2013.
[5] N. Bharathi, C. M. Ramakritinan, and K. Kolandaswamy, "Geographical information system based study on Dengue and Chikungunya at Western Ghats districts, Tamil Nadu, India," Int. J. Mosq. Res. IJMR, vol. 2, no. 23, pp. 30-38, 2015.

R. Carter, "Spatial simulation of malaria transmission and its contro by malaria transmission blocking vaccination," Int. J. Parasitol., 2002. A. Chakravarti and R. Kumaria, "Eco-epidemiological analysis of dengue infection during an outbreak of Dengue Fever, India," Virol. J., vol. 2, 2005.

[8] A. Earnest and S. Tan, "Meteorological factors and El Nino Southern Oscillation are independently associated with dengue infections," Epidemiol., 2012.

[9] G. Killeen, B. Knols, and W. Gu, "Taking malaria transmission out of the bottle: implications of mosquito dispersal for vector-control interventions," Lancet Infect. Dis., 2003.

[10] H. Thu, K. Aye, and S. Thein, "The effect of temperature and humidity on dengue virus propagation in Aedes aegypti mosquitos.," 1998.

[11] Z. Xu, W. Blot, H. Xiao, and A. Wu, "Smoking, air pollution, and the high rates of lung cancer in Shenyang, China," J., 1989.

[12] K. Krivoruchko, "Empirical bayesian kriging," Esri Redlands, CA, USA, 2012.

[13] J. S. Coleman, "Introduction to Mathematical Sociology.," 1964.

[14] Y. Cheong, K. Burkart, P. Leitão, and T. Lakes, "Assessing weather effects on dengue disease in Malaysia," Int. J., 2013.

R. Dhiman, S. Pahwa, G. Dhillon, and A. Dash, "Climate change and threat of vector-borne diseases in India: are we prepared?," Parasitol. Res., 2010.

[16] P. Jeefoo, N. Tripathi, and M. Souris, "Spatio-temporal diffusion pattern and hotspot detection of dengue in Chachoengsao province, Thailand," Int. J. Environ., 2010.

[17] S. A. Agarwal, S. S. Sikarwar, and D. Sukumaran, "Application of RS \&amp; GIS in risk area assessment for mosquito borne diseases-a case study in a part of Gwalior," Int J Adv. Technol Eng Res , pp. 1-4, 2012.

[18] M. Palaniyandi, "Web mapping GIS : GPS under the GIS umbrella for Aedes species dengue and chikungunya vector mosquito surveillance and control," Int. J. Mosq. Res., vol. 1, no. 3, pp. 18-25, 2014.

[19] A. Srivastava, B. Nagpal, and R. Saxena, "Malaria epidemicity of Mewat region, District Gurgaon, Haryana, India: a GIS-based study," Curr. Sci., 2004.

[20] S. Coxe, S. West, and L. Aiken, "The analysis of count data: A gentle introduction to Poisson regression and its alternatives," J. Pers. Assess., 2009.

[21] M. Johansson, F. Dominici, and G. Glass, "Local and global effects of climate on dengue transmission in Puerto Rico," PLoS Negl Trop Dis, 2009.

[22] J. Patz, W. Martens, and D. Focks, "Dengue Fever epidemic potential as projected by general circulation models of global climate change.," Environ. Heal., 1998.

[23] CDC, "Mosquito life-cycle. Dengue Homepage. Atlanta: USA Government," 2012.

[24] Y. Hii, "Climate and Dengue Fever: early warning based on temperature and rainfall," 2013.

[25] A. Rohani, Y. Wong, I. Zamre, and H. Lee, "The effect of extrinsic incubation temperature on development of dengue serotype 2 and 4 viruses in Aedes aegypti (L.)," Asian J. ..., 2009.

[26] H. M. Yang, M. L. G. Macoris, K. C. Galvani, M. T. M. Andrighetti, and D. M. V Wanderley, "Assessing the effects of temperature on the population of Aedes aegypti, the vector of dengue.," Epidemiol. Infect., vol. 137, no. 8, pp. 1188-202, Aug. 2009.

[27] C. Depradine and E. Lovell, "Climatological variables and the incidence of Dengue Fever in Barbados," Int. J. Environ., 2004.

[28] J. Yasuoka and R. Levins, "Ecology of vector mosquitoes in Sri Lanka--suggestions for future mosquito control in rice ecosystems.," 2007.

[29] L. Lu, H. Lin, L. Tian, W. Yang, J. Sun, and Q. Liu, "Time series analysis of Dengue Fever and weather in Guangzhou, China," $B M C$ Public Health, vol. 9, no. 1, p. 395, Dec. 2009.

[30] D. Rogers, "Dengue: recent past and future threats," Phil. Trans. $R$ Soc. B, 2015.

[31] D. Rogers and S. Randolph, "Climate change and vector-borne diseases," Adv. Parasitol., 2006.

About the Authors:

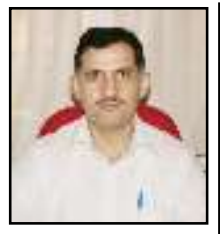

Dr. Mahesh Kumar Jat

Dr. Jat is presently working as an Associate Professor in Civil Engineering Department, Malaviya National Institute of Technology Jaipur (India). His research interests are Integrated Water Resources Management and Geo-spatial Technologies.

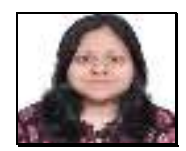

Miss Shuchi Mala

She is currently pursuing $\mathrm{PhD}$ from Civil Engineering Department, Malaviya National Institute of Technology Jaipur. 